\title{
Analysis of differences in exercise recognition by constraints on physical activity of hospitalized cancer patients based on their medical history
}

\author{
Mi-Ri Choi', Sang-Wan Jeon², Eun-Surk Yi ${ }^{1}{ }^{1} *$ \\ 'Department of Exercise Rehabilitation \& Welfare, College of Health Science, Gachon University, Incheon, Korea \\ ${ }^{2}$ Exercise Rehabilitation Research Institute, Gachon University, Incheon, Korea
}

The purpose of this study is to analyze the differences among the hospitalized cancer patients on their perception of exercise and physical activity constraints based on their medical history. The study used questionnaire survey as measurement tool for 194 cancer patients (male or female, aged 20 or older) living in Seoul metropolitan area (Seoul, Gyeonggi, Incheon). The collected data were analyzed using frequency analysis, exploratory factor analysis, reliability analysis $t$-test, and oneway distribution using statistical program SPSS 18.0. The following results were obtained. First, there was no statistically significant difference between cancer stage and exercise recognition/physical activity constraint. Second, there was a significant difference between cancer stage and sociocultural constraint/facility constraint/program constraint. Third, there was a significant difference between cancer operation history and physical/socio-cultural/facility/program constraint.
Fourth, there was a significant difference between cancer operation history and negative perception/facility/program constraint. Fifth, there was a significant difference between ancillary cancer treatment method and negative perception/facility/program constraint. Sixth, there was a significant difference between hospitalization period and positive perception/negative perception/physical constraint/cognitive constraint. In conclusion, this study will provide information necessary to create patient-centered healthcare service system by analyzing exercise recognition of hospitalized cancer patients based on their medical history and to investigate the constraint factors that prevents patients from actually making efforts to exercise.

Keywords: Exercise recognition, Physical activity, Medical history, Cancer, Constraints, Analysis of differences

\section{INTRODUCTION}

As cancer patients are increasing at an annual rate of $10 \%$, cancer is the highest cause of death for last 33 years in Korea (Statistics Korea, 2016). American College of Sports Medicine recommends for cancer patients to engage in following physical activities: $150 \mathrm{~min}$ of medium to high intensity physical activity a week, 75 min of high intensity physical activities in addition to whole body large muscle training for at least twice a week. However, the most of cancer patients have low physical activity compared to healthy people. Injury to organs by cancer or the effect of chemotherapy restricts movement. Fatigue, declined physical strength, and unfit physical condition cause restriction in exercise

participation (Ray et al., 2008). Cancer patients are in a lower physical and mental condition due to pain induced by chemotherapy and other treatments. They are in a state where they do not even have a will to physical exercise.

In order to improve this situation, various physical activity programs developed and applied both in Korea and abroad (George et al., 2014; Schmitz et al., 2010; Speck et al., 2010; Velthuis et al., 2010). However, most of programs focused on temporary physical trainings, therefore there was a limitation to develop sustained exercise. In the cases of overseas, various studies have been done to develop environment-centered public health service to stimulate physical activity of patients at hospital (Drahota et al., 2012). Studies on physical activity for cancer patients both at

${ }^{*}$ Corresponding author: Eun-Surk Yi iD https://orcid.org/0000-0002-9370-5231 Department of Exercise Rehabilitation \& Welfare, College of Health Science, Gachon University, 191 Hambangmoe-ro, Yeonsu-gu, Incheon 21936, Korea Tel: +82-32-820-4442, Fax: +82-32-820-4449, E-mail: Yies@gachon.ac.kr Received: March 3, 2018 / Accepted: April 2, 2018 
home and abroad continued, and the most of these studies have demonstrated that physical activity has a positive effect on treatment and reduces side effect of chemotherapy.

In conclusion, the researches on the subject of physical activities of cancer patients done so far have a limitation of not having investigate from multiple angles pathological conditions and other factors, e.g., cancer diagnosis, cancer operation, recurrence, ancillary treatment. Analyzing the medical condition of cancer patients and the recognizing and performing physical activity of cancer patients can create substantial value for patient's recovery. Therefore, it is meaningful to analyze the hospital environment and individual patient characteristics according to pathological history of cancer patients and finding the restricting factor that limits their physical activity.

\section{MATERIALS AND METHODS}

\section{Research subjects}

The research defined male/female cancer patients aged 20 years or older living in Seoul metropolitan area (Seoul, Gyeonggi-do, Incheon) as population sample. Then purposive sampling was used to select 230 cancer patients at general hospitals located in the metropolitan area. The survey period was from March to May, 2017. Data from 36 persons that contained omission and invalid answers were dropped and the data for final 194 persons were used for analysis.

Data are as follows. Gender: 72 men (37.1\%), 122 women (62.9\%). Age: 3 persons in their 20s (1.5\%), 12 persons in their 30 s $(6.2 \%), 39$ persons in their 40 s $(20.1 \%), 67$ persons in their $50 \mathrm{~s}(34.5 \%), 39$ persons in their $60 \mathrm{~s}$ (39.1\%), 25 persons in their 70 s $(12.9 \%), 9$ persons in their 80 s (4.6\%). Education: 51 middle school graduate (26.3\%), 93 high school graduate (47.9\%), 39 university graduate (20.1\%), 11 graduate school graduate $(5.7 \%)$. Income: 72 persons with 2 million won or lower (37.1\%), 61 persons between 2 million and 4 million won (31.4\%), 41 persons between 4 million and 6 million won (21.1\%), 7 persons between 6 million and 8 million won (3.6\%), 2 persons between 8 million and 10 million won (1.0\%), 11 persons 10 million or above 95.7\%). Marital status: 164 persons married (84.5\%), 17 persons unmarried (8.9\%), 13 persons others $(6.7 \%)$.

\section{Research method}

Questionnaires were used to analyze the differences in subjective evaluation of health condition, exercise recognition and personal constraints according to the medical history of hospitalized cancer patients. The questionnaire on exercise awareness decision balance developed by Nigg et al. (2001). The questionnaires on physical activity constraints were created by revising and supplementing the questionnaires developed by Zhu et al. (2001) to fit the contents of this research. Preliminary questionnaires were performed for 50 hospitalized cancer patients. The finalized questionnaire consisted of the followings: 5 questions on background variables, 6 questions on medical condition, 10 questions on exercise awareness, 8 questions on environmental constraints, and 13 questions on persona constraints.

\section{Reliability and validity of questionnaire survey}

An expert group consisting of mainly doctorate degree holders in nursing, physical therapy, exercise rehabilitation, medical science, sports sociology, and exercise physiology was asked to review and evaluate the questionnaire used in this research, where they discussed the validity of contents and the appropriateness of questions. Based on the data collected, confirmatory factor analysis was used to perform final validation. Principal component analysis was used to extract principal factors and varimax technique based on orthogonal rotation method was selected to simplify factor loading. Kaiser-Meyer-Olkin (KMO) measure was used for variables to be subject to factor analysis. The research used the Bartlett's test of sphericity coefficient $\left(\chi^{2}\right)$, which indicates the appropriateness of applying factor analysis and common factors. In terms of the selection criteria for questions, only the factors extracted with eigenvalue of 1.0 and above were used. The results of exploratory factor analysis are as follows: for exercise awareness, $\mathrm{KMO}=0.890$, $\chi^{2}=1,083.397$; the eigenvalue of positive recognition and negative recognition were 5.044 and 1.170, respectively; for environmental physical activity constraints, $\mathrm{KMO}=0.668, \chi^{2}=434.496$; the eigenvalues of facilities and programs were 2.522 and 1.889; for individual physical activity constraints, $\mathrm{KMO}=0.622, \chi^{2}=$ 535.166; the eigenvalue of cognitive psychology, social culture, and physical body were $2.103,2.030$, and 1.843 .

Cronbach a coefficient was used to reliability analysis, and the criteria for factor loading value was set at 0.05 and above. As result, the 4th question in individual exercise constraints was removed, while all other factors obtained validity and reliability coefficient (positive recognition $=0.927$, negative recognition $=0.661$, facility $=0.657$, program $=0.709$, cognitive psychology $=0.783$, social culture $=0.731$, body $=0.675$ ).

\section{Handling resources}

The purpose of this study is to investigate whether there is a 
difference between subjective health condition, exercise awareness and individual exercise constraints according to the medical history of hospitalized cancer patients. To conduct this research, the researcher and the assistants visited hospital in person to do questionnaire survey after obtaining approval from the hospital. The patients participating in the research were explained the purpose of research and how to complete the questionnaire. The purpose of the questionnaire and the method of business were explained to the survey subjects. They were then asked to complete the questionnaire using self-reporting method, and their answers were collected. Then, the answers deemed to have low reliability and the answers that contained double entry or no-entry were omitted from analysis. The data that were considered reliable were input (coded) to computer individually. IBM SPSS ver. 18.0 (IBM Co., Armonk, NY, USA) was used to handle the work according to the purpose of analysis. For statistical analysis, descriptive statistical

Table 1. Differences in health awareness and physical activity constraints among cancer patients according to their diagnosed cancer

\begin{tabular}{lccc}
\hline Category & $\begin{array}{c}\text { Major cancers } \\
(\mathrm{n}=146)\end{array}$ & $\begin{array}{c}\text { Other cancers } \\
(\mathrm{n}=48)\end{array}$ & $t$ \\
\hline Positive exercise awareness & $4.05 \pm 0.649$ & $3.87 \pm 0.809$ & 1.464 \\
Negative exercise awareness & $2.59 \pm 0.842$ & $2.44 \pm 0.783$ & 0.191 \\
Physical constraint & $2.87 \pm 1.133$ & $3.05 \pm 1.447$ & -0.831 \\
Cognitive psychological constraint & $2.28 \pm 0.676$ & $2.09 \pm 0.762$ & 1.660 \\
Socio-cultural constraint & $2.90 \pm 0.747$ & $2.86 \pm 0.962$ & 0.268 \\
Facility constraint & $3.22 \pm 0.758$ & $3.30 \pm 0.565$ & -0.822 \\
Program constraint & $3.35 \pm 0.937$ & $3.52 \pm 0.818$ & -1.238 \\
\hline
\end{tabular}

Values are presented as mean \pm standard deviation.

Major cancers: gastric cancer, breast cancer, thyroid cancer, uterine cancer, lung cancer, colorectal cancer, liver cancer. Other cancers: skin fibrosarcoma, spinal cancer, brain adenocarcinoma, lymphoma, head and neck cancer, parental cancer, brain cancer, blood cancer, ovarian cancer, pancreatic cancer, osteosarcoma, myeloma, gallbladder cancer, biliary cancer, rectal cancer, leukemia, esophageal cancer. analysis, exploratory factor analysis, $t$-test and one-way analysis of variance (ANOVA) were used.

\section{RESULTS}

\section{Difference between exercise recognition and physical activity constraint based on cancer diagnosis}

As shown in Table 1, $t$-test was conducted to determine the difference between exercise awareness and physical activity constraint according to cancer diagnosis. The analysis of the effects of exercise awareness and physical activity constraints on diagnosis showed that the difference was not statistically significant. However, positive exercise awareness was highest among cancer patients (major cancers [mean \pm standard deviation, $4.05 \pm 0.649$ ] and other cancers [3.87 \pm 0.809$]$ ), from which it can be inferred that they regarded exercise as positive thing.

\section{Differences in exercise awareness and physical activity constraints according to diagnosis}

As shown in Table 2, one-way ANOVA was performed to determine the differences in exercise awareness and physical activity constraints according to cancer stage. The analysis of the effect of exercise awareness and physical activity constraints according to cancer stage showed statistically significant difference in socio-cultural constraints $(F=3.358)$, facility constraints $(F=2.684)$, and program constraints $(F=9.217)$. In particular, difference in program constraint according to cancer stage showed highest statistical significance $(P<0.001)$.

Posttest (Tukey) was performed to analyze the group differences. Physical activity constraints were higher in stage 2 group than stage 1 and 4 groups in terms of socio-cultural constraints; and stage 3 group is higher than sage 0 group in terms of facility con-

Table 2. Differences in health awareness and physical activity constraints according to cancer stage of hospitalized constraints

\begin{tabular}{|c|c|c|c|c|c|c|c|c|}
\hline Category & Stage $0(n=5)$ & Stage $1(n=20)$ & Stage $2(n=42)$ & Stage $3(n=57)$ & Stage $4(n=22)$ & $\begin{array}{l}\text { Unknown } \\
(n=48)\end{array}$ & $F$ & Tukey \\
\hline Positive exercise awareness & $3.96 \pm 0.219$ & $3.96 \pm 0.705$ & $4.20 \pm 0.620$ & $3.99 \pm 0.685$ & $3.90 \pm 0.872$ & $3.86 \pm 0.758$ & 1.125 & \\
\hline Negative exercise awareness & $1.72 \pm 0.264$ & $2.14 \pm 0.790$ & $2.24 \pm 0.683$ & $2.37 \pm 0.776$ & $2.39 \pm 0.830$ & $2.46 \pm 0.652$ & 1.874 & \\
\hline Physical constraints & $1.92 \pm 0.395$ & $3.10 \pm 1.022$ & $3.03 \pm 1.188$ & $2.95 \pm 1.332$ & $2.84 \pm 1.087$ & $2.84 \pm 1.314$ & 1.461 & \\
\hline Cognitive psychology constraints & $1.78 \pm 0.441$ & $2.23 \pm 0.548$ & $2.18 \pm 0.638$ & $2.38 \pm 0.638$ & $1.98 \pm 0.686$ & $2.33 \pm 0.831$ & 2.157 & \\
\hline Socio-cultural constraints & $2.89 \pm 0.957$ & $2.45 \pm 0.694$ & $3.13 \pm 0.721$ & $2.94 \pm 0.784$ & $2.53 \pm 1.067$ & $3.00 \pm 0.581$ & $3.358^{* *}$ & $\mathrm{~b}<\mathrm{e}<\mathrm{c}$ \\
\hline Facility constraints & $2.67 \pm 0.545$ & $3.10 \pm 0.644$ & $3.29 \pm 0.647$ & $3.42 \pm 0.762$ & $3.13 \pm 0.640$ & $3.10 \pm 0.756$ & $2.684^{*}$ & $a<d$ \\
\hline Program constraints & $2.28 \pm 0.263$ & $2.91 \pm 1.141$ & $3.18 \pm 0.810$ & $3.70 \pm 0.723$ & $3.93 \pm 0.821$ & $3.306 \pm 0.906$ & $9.217^{* * *}$ & $\mathrm{a}<\mathrm{c}<\mathrm{d}<\mathrm{e}$ \\
\hline
\end{tabular}

Values are presented as mean \pm standard deviation.

a, stage $0(n=5) ;$, stage $1(n=20) ; c$, stage $2(n=42) ; d$, stage $3(n=57) ;$ e, stage $4(n=22) ; f$, unknown $(n=48)$.

${ }^{*} P<0.05$. ${ }^{* *} P<0.01$. ${ }^{* *} P<0.001$. 
straints. Physical activity constraints were higher in stage 4 group than stage 3, 2, 0 groups in terms of program constraints.

\section{Difference between exercise recognition and physical activity constraint based on operation history}

As shown in Table 3, $t$-test was performed to determine the difference between exercise awareness and physical activity constraints according to cancer operation history. The analysis of the effect of exercise awareness and physical activity constraints according to cancer operation history shows statistically significant differences in physical constraints $(t=-2.801)$, socio-cultural constraint $(t=2.700)$, facility constraint $(t=-3.678)$, and program constraints ( $t=-3.678)$. In particular, physical activity constraints were higher in operation group in terms of physical and socio-cultural constraints; nonoperation group in terms of facility constraints and program constraints.

\section{Difference between exercise recognition and physical activity constraint based on recurrence of cancer}

As shown in Table 4, $t$-test was conducted to determine the dif-

Table 3. Differences in health awareness and physical activity constraints according to cancer operation history of hospitalized cancer patients

\begin{tabular}{lccc}
\hline Category & Yes $(n=140)$ & No $(n=54)$ & $t$ \\
\hline Positive exercise awareness & $3.95 \pm 0.604$ & $4.23 \pm 0.923$ & -1.892 \\
Negative exercise awareness & $2.44 \pm 0.805$ & $2.92 \pm 0.809$ & -1.305 \\
Physical constraints & $3.04 \pm 1.251$ & $2.46 \pm 0.973$ & $2.801^{* *}$ \\
Cognitive psychology constraints & $2.23 \pm 0.657$ & $2.26 \pm 0.847$ & -0.259 \\
Socio-cultural constraints & $2.97 \pm 0.749$ & $2.60 \pm 0.924$ & $2.700^{* *}$ \\
Facility constraints & $3.14 \pm 0.668$ & $3.58 \pm 0.775$ & $-3.680^{* * *}$ \\
Program constraints & $3.26 \pm 0.909$ & $3.83 \pm 0.778$ & $-3.678^{* * *}$ \\
\hline
\end{tabular}

Values are presented as mean \pm standard deviation.

${ }^{* *} P<0.01$. ${ }^{* *} P<0.001$ ference between exercise awareness and physical activity constraints according to the status of cancer recurrence. The analysis of the effect of exercise awareness and physical activity constraints according to the status of cancer recurrence shows statistical significance for negative perception of exercise $(t=2.571)$, facility constraints $(t=2.162)$, and program constraints $(t=3.861)$. For cancer recurrence group, negative perception of exercise (mean, 2.56) was higher than nonrecurrence group. Also, for program constraints (mean, 3.79) and facility constraints (mean, 3.41), physical activity constraints were higher in cancer recurrence group than nonrecurrence group.

\section{Difference between exercise awareness and physical activity constraint based on supplementary treatment method}

As shown in Table 5, one-way ANOVA was performed to determine the difference between exercise awareness and physical activity constraints according to supplementary treatment methods. The analysis of the effect of supplementary treatment method according to exercise awareness and physical activity constraints

Table 4. Differences in health awareness and physical activity constraints according to the recurrence of cancer of hospitalized cancer patients

\begin{tabular}{lccc}
\hline Category & Yes $(n=39)$ & No $(n=155)$ & $t$ \\
\hline Positive exercise awareness & $3.97 \pm 0.744$ & $4.02 \pm 0.684$ & -0.390 \\
Negative exercise awareness & $2.56 \pm 0.753$ & $2.23 \pm 0.721$ & $2.571^{*}$ \\
Physical constraints & $3.18 \pm 1.428$ & $2.84 \pm 1.150$ & 1.590 \\
Cognitive psychology constraints & $2.11 \pm 0.775$ & $2.27 \pm 0.680$ & -1.288 \\
Socio-cultural constraints & $2.95 \pm 0.726$ & $2.88 \pm 0.824$ & 0.514 \\
Facility constraints & $3.41 \pm 0.483$ & $3.19 \pm 0.759$ & $2.162^{*}$ \\
Program constraints & $3.79 \pm 0.669$ & $3.29 \pm 0.937$ & $3.861^{* * *}$
\end{tabular}

Values are presented as mean \pm standard deviation ${ }^{*} P<0.05$. ${ }^{* *} P<0.001$

Table 5. Differences in health awareness and physical activity constraints according to supplementary treatment method of hospitalized cancer patients

\begin{tabular}{lccccccc}
\hline Category & Chemo $(n=97)$ & Radiation $(n=4)$ & $\begin{array}{c}\text { Chemo+radiation } \\
(n=31)\end{array}$ & Others $(n=35)$ & None $(n=27)$ & $F$ & Tukey \\
\hline Positive awareness & $4.01 \pm 0.584$ & $3.78 \pm 0.851$ & $4.22 \pm 0.764$ & $3.99 \pm 0.737$ & $3.78 \pm 0.805$ & 1.691 \\
Negative awareness & $2.33 \pm 0.815$ & $2.32 \pm 0.681$ & $2.61 \pm 0.677$ & $2.04 \pm 0.526$ & $2.07 \pm 0.657$ & $3.399^{*}$ & $\mathrm{~d}<\mathrm{c}$ \\
Physical constraints & $3.04 \pm 1.207$ & $3.39 \pm 1.872$ & $2.59 \pm 1.310$ & $2.89 \pm 0.893$ & $2.70 \pm 1.106$ & 1.454 \\
Cognitive psychology constraints & $2.14 \pm 0.683$ & $2.42 \pm 0.990$ & $2.49 \pm 0.841$ & $2.23 \pm 0.539$ & $2.11 \pm 0.487$ & 2.009 \\
Socio-cultural constraints & $2.83 \pm 0.899$ & $3.21 \pm 0.583$ & $2.76 \pm 0.674$ & $3.07 \pm 0.690$ & $2.92 \pm 0.816$ & 1.313 \\
Facility constraints & $3.48 \pm 0.672$ & $3.59 \pm 0.645$ & $3.13 \pm 0.731$ & $2.74 \pm 0.564$ & $3.06 \pm 0.612$ & $9.825^{* * *}$ \\
Program constraints & $3.50 \pm 0.957$ & $3.50 \pm 0.387$ & $3.63 \pm 0.731$ & $2.85 \pm 0.860$ & $3.38 \pm 0.961$ & $4.470^{* *}$ & $\mathrm{~d}<\mathrm{c}<<\mathrm{b}$ \\
\hline
\end{tabular}

Values are presented as mean \pm standard deviation.

a, chemo $(n=97)$; b, radiation $(n=4)$; c chemo+radiation $(n=31)$; $d$, others $(n=35)$; e, none $(n=27)$.

${ }^{*} P<0.05 .{ }^{* *} P<0.01 .{ }^{* * *} P<0.001$. 
show statistically significant difference in negative perception of exercise $(F=3.399)$, facility constraints $(F=9.825)$ and program constraints $(F=4.470)$. In particular, program constraints had the most statistically significant difference in terms of supplementary treatment $(P<0.001)$. Especially, the statistically significant difference was found between the facility restriction and the supplementary treatment method $(P<0.001)$. A posttest (Tukey) was performed to analyze group differences. In terms of negative perception of exercise, the group receiving both chemotherapy and radiation therapy had higher negative perception of exercise. Moreover, physical activity constrains were higher in radiation therapy group than other or chemo+radiation therapy group in terms of facility constraints; and higher in chemo+radiation therapy group in terms of program constraints.

\section{Difference between exercise awareness and physical activity constraint based on hospitalization period}

As shown in Table 6, one-way ANOVA analysis was performed to analyze the differences between exercise awareness and physical activity constraints according to hospitalization period. The analysis of the effect of exercise awareness and physical activity constraints according to hospitalization period shows statistically significant differences in positive awareness $(F=8.690)$, negative awareness $(F=3.655)$, physical activity constraints $(F=4.243)$ and cognitive psychological constraints $(F=3.868)$. In particular, positive awareness showed the highest statistical difference according to hospitalization period $(P<0.001)$.

A postanalysis (Tukey) was performed to analyze group differences. For positive awareness, the group with 7 weeks or lower and the group with longer than 1 week but less than 2 weeks had higher positive awareness compared to the group with 2 weeks or more. For negative awareness, the group with 2 weeks or longer had higher negative awareness than the group 1 week or longer but less than 2 weeks. For physical activity constraints, the group with 2 weeks or more was higher than other groups. For cognitive psychology constraint, the group with 1 week or more but less than 2 weeks was higher than the group with 2 weeks or more in terms of physical activity constraints.

\section{DISCUSSION}

The aim of this research was to conduct empirical study of the difference between exercise awareness and physical activity constraints of cancer patients and tried to understand the difference according to medical history of patients (diagnosis, cancer stage, operation history, recurrence history, supplementary treatment method, and hospitalization period).

First, diagnosed cancer types did not show statistically significant difference between exercise awareness and physical activity constraints. Physical activities has a good effect on adult cancer patients and positive effect on exercise motivation (Midtgaard et al., 2009). It stimulates the exercise awareness of cancer patients and minimize physical activity constraints, helping them engage in physical activities more actively. With recent increase in cancer survival rate, there is an increasing interest in the health and the quality of life of cancer survivors. In particular, the awareness about the importance of exercise is increased as exercise is known to have positive effect on the survival rate, immune function and the quality of life of cancer survivors. However, according to the results of this study, there was no statistically significant difference in perceived exercise awareness and physical activity constraint between major cancer patients and other cancer patients. From this, it can be inferred that most research subjects being hospitalized cancer patients receiving chemotherapy or surgery operation, they are not in a situation to pay attention to exercises and physical activity constraints in reality.

Table 6. Differences in health awareness and physical activity constraints according to hospitalization period of cancer patients

\begin{tabular}{|c|c|c|c|c|c|}
\hline Category & 7 Days or less $(n=118)$ & Between $1-2$ weeks ( $n=28$ ) & 2 Weeks or more $(n=24)$ & $F$ & Tukey \\
\hline Positive awareness & $4.09 \pm 0.629$ & $4.11 \pm 0.834$ & $3.53 \pm 0.693$ & $8.690^{* * *}$ & $c<a, b$ \\
\hline Negative awareness & $2.33 \pm 0.754$ & $1.96 \pm 0.564$ & $2.47 \pm 0.731$ & $3.655^{*}$ & $b<c$ \\
\hline Physical constraints & $2.82 \pm 1.218$ & $2.71 \pm 1.017$ & $3.50 \pm 1.240$ & $4.243^{*}$ & $a, b<c$ \\
\hline Cognitive psychology constraints & $2.21 \pm 0.712$ & $2.05 \pm 0.609$ & $2.54 \pm 0.657$ & $3.868^{*}$ & $b<c$ \\
\hline Socio-cultural constraints & $2.87 \pm 0.846$ & $3.05 \pm 0.678$ & $2.82 \pm 0.693$ & 0.660 & \\
\hline Facility constraints & $3.26 \pm 0.708$ & $3.07 \pm 0.611$ & $3.28 \pm 0.830$ & 0.863 & \\
\hline Program constraints & $3.43 \pm 0.949$ & $3.23 \pm 0.765$ & $3.34 \pm 0.846$ & 0.553 & \\
\hline
\end{tabular}

Values are presented as mean \pm standard deviation.

a, 7 days or less $(n=118)$; b, between $1-2$ weeks $(n=28) ;$ c, 2 weeks or more $(n=24)$.

${ }^{*} P<0.05$. ${ }^{* *} P<0.001$. 
Second, there was a statistically significant difference in cancer stage for program constraint, facility constraint and socio-cultural constraint among the factors of exercise awareness and physical activity constraints. According to the prior researches done on the subject, lack of exercise information could limit cancer patients receiving chemotherapy in engaging in exercise (Adamsen et al., 2004). The exercise information supplied by hospital is limited to "appropriate" level, according to the report, but there is severe limitation on implementation in reality (Lin et al., 2014). This result partially supports the conclusion of this research. In particular, the more severe stage the patient group is in, the higher the desire for recovery and the perceived necessity of exercise. However, since the "appropriate" programs fall below the expectation of patients, the level of environmental constraint seems to increase.

Third, cancer operation history has a statistically significant difference in terms of physical constraint, socio-cultural constraint, facility constraint and program constraint. To be specific, the group with cancer operation history had higher physical activity constraint and socio-cultural constraint compared to the group without operation history. Some prior researches reported that colorectal cancer patients receiving chemotherapy after cancer surgery tend to experience physical and emotional constraints as side effect of treatment (Chung et al., 2013), which corroborates the result of this research. In particular, the patients who received cancer surgery limited hospital life and immediate disability in carrying out ordinary life bring physiological changes such as decline in physical strength and reduction in muscle, affecting not only physical constraint but also socio-cultural constraint. On the other hand, in the noncancer operation group, the difficulty in physical mobility is less than the operation group, which leads to expectation and participation of the facility and the program, magnifying the feeling of constraint.

Fourth, cancer occurrence had a statistically significant difference in terms of negative awareness, facility constraint and program constraint. In general, when cancer recurs, most patients have not completely escaped from cancer, and they could be proactively seeking various treatment methods. In particular, with the release of case studies claiming that physical activities could reduce recurrence of cancer and help recovery, patients have developed a positive view of physical activities (Meyerhardt et al., 2006). However, as shown in this research, cancer recurrence could create negative perception about exercise despite the fact that physical activities are effective for cancer prevention and management. On the other hand, cancer patients tend to remain immobile when cancer recurs or during treatment in the face of pain and psychological weakening (Fadul et al., 2008). In particular, exercise facilities at hospital or the absence of program could accelerate physical activity constraints.

Fifth, supplementary treatment methods showed statistically significant differences in negative awareness, facility constraints, and program constraints. Cancer patients were perceiving as physical activity constraints during the chemotherapy treatment stage lack of information on exercise and wanted to receive information on customized exercise methods and that consider various side effects (Adamsen et al., 2009). However, most exercise-related activities in the hospital do not exceed the level of temporary exercise. This is consistent with the results of this study and can be interpreted as a result of the need for a systematic exercise system that can be continuously managed and operated. On the other hand, radiotherapy and chemotherapy treatments appear to make patients feel resistance against physical activities by causing physical and mental pain.

Sixth, hospitalization period showed statistically significant difference in positive awareness, negative awareness, physical constraint, and cognitive psychological constraint. When hospitalization period exceeds 2 weeks, positive awareness of exercise decreases while negative awareness increases, which indicates that an increase in the length of hospitalization adversely affects the awareness of physical activities. In addition, physical restraint also showed that the longer the hospitalization period, the more physical activity constraints in the hospital becomes.

It was reported that cancer diagnosis leads to increased awareness of health management and then to physical activities, resulting in higher physical activities than that of the adult in their middle age (Liou et al., 2011). Likewise, despite high demand for physical activities for recovery, most hospitals lack systematic program for physical activities, and the place needed for such program is not in priority order. At the end, it can be seen that the environment for the physical activity of the patients is relatively insufficient, which affects their exercise awareness too. In recent years, there has been a rapid increase in evidence for the beneficial effects of physical activity on cancer, and patient's physical activities received much attention as non-pharmaceutical alternatives to hospitalized cancer patients. However, it should be noted that the effect could vary according to patient's condition, hospital system, and environment.

In conclusion, the stage of cancer of hospitalized cancer patients, the history of cancer operation surgery, recurrence, supplementary treatment methods, and the hospitalization period may partially affect cancer patients' exercise awareness and physical ac- 
tivity constraints. Therefore, it is necessary to pay special attention to the cancer patient's physical activities when reviewing his/her medical history. At the end, improving individual and environmental constraints for the cancer patient's intervention treatment for physical activities may play a significant role in patient recovery. In conclusion, it is necessary to develop questionnaire survey that can categorize and analyze in depth the medical history of hospitalized cancer patients and continue to conduct researches to improve exercise awareness and help patients improve constraints they face at hospital from the perspective of public health service.

\section{CONFLICT OF INTEREST}

No potential conflict of interest relevant to this article was reported.

\section{ACKNOWLEDGMENTS}

This work was supported by the Ministry of Education of the Republic of Korea and the National Research Foundation of Korea (NRF-2016S1A5B6913737).

\section{REFERENCES}

Adamsen L, Midtgaard J, Andersen C, Quist M, Moeller T, Roerth M. Transforming the nature of fatigue through exercise: qualitative findings from a multidimensional exercise programme in cancer patients undergoing chemotherapy. Eur J Cancer Care (Engl) 2004;13:362-370.

Adamsen L, Quist M, Andersen C, Møller T, Herrstedt J, Kronborg D, Baadsgaard MT, Vistisen K, Midtgaard J, Christiansen B, Stage M, Kronborg MT, Rørth M. Effect of a multimodal high intensity exercise intervention in cancer patients undergoing chemotherapy: randomised controlled trial. BMJ 2009;339:b3410.

Chung JY, Lee DH, Park JH, Lee MK, Kang DW, Min J, Kim DI, Jeong DH, Kim NK, Meyerhardt JA, Jones LW, Jeon JY. Patterns of physical activity participation across the cancer trajectory in colorectal cancer survivors. Support Care Cancer 2013;21:1605-1612.

Drahota A, Ward D, Mackenzie H, Stores R, Higgins B, Gal D, Dean TP. Sensory environment on health-related outcomes of hospital patients. Cochrane Database Syst Rev 2012;3:CD005315.

Fadul NA, El Osta B, Dalal S, Poulter VA, Bruera E. Comparison of symptom burden among patients referred to palliative care with hematologic malignancies versus those with solid tumors. J Palliat Med 2008; 11:422-427.
George SM, Alfano CM, Groves J, Karabulut Z, Haman KL, Murphy BA, Matthews CE. Objectively measured sedentary time is related to quality of life among cancer survivors. PLoS One 2014;9:e87937.

Lin KY, Shun SC, Lai YH, Liang JT, Tsauo JY. Comparison of the effects of a supervised exercise program and usual care in patients with colorectal cancer undergoing chemotherapy. Cancer Nurs 2014;37:E2129 .

Liou YM, Lee HL, Chien LY, Kao WY, Chiang CC, Wang DY. Daily-life physical activity and related factors among patients with cancer receiving chemotherapy in Taiwan. Cancer Nurs 2011;34:443-452.

Meyerhardt JA, Heseltine D, Niedzwiecki D, Hollis D, Saltz LB, Mayer RJ, Thomas J, Nelson H, Whittom R, Hantel A, Schilsky RL, Fuchs CS. Impact of physical activity on cancer recurrence and survival in patients with stage III colon cancer: findings from CALGB 89803. J Clin Oncol 2006;24:3535-3541.

Midtgaard J, Baadsgaard MT, Møller T, Rasmussen B, Quist M, Andersen C, Rørth M, Adamsen L. Self-reported physical activity behaviour; exercise motivation and information among Danish adult cancer patients undergoing chemotherapy. Eur J Oncol Nurs 2009;13:116-121.

Nigg CR, Norman GJ, Rossi JS, Benisovich SV. Examining the structure of physical self-description using an American university sample. Res Q Exerc Sport 2001;72:78-83.

Ray M, Rogers LQ, Trammell RA, Toth LA. Fatigue and sleep during cancer and chemotherapy: translational rodent models. Comp Med 2008;58:234-245.

Schmitz KH, Courneya KS, Matthews C, Demark-Wahnefried W, Galvão DA, Pinto BM, Irwin ML, Wolin KY, Segal RJ, Lucia A, Schneider CM, von Gruenigen VE, Schwartz AL; American College of Sports Medicine. American College of Sports Medicine roundtable on exercise guidelines for cancer survivors. Med Sci Sports Exerc 2010;42:14091426.

Speck RM, Courneya KS, Mâsse LC, Duval S, Schmitz KH. An update of controlled physical activity trials in cancer survivors: a systematic review and meta-analysis. J Cancer Surviv 2010;4:87-100.

Statistics Korea. Cause of death for Koreans. Daejeon: Statistics Korea; 2016.

Velthuis MJ, Agasi-Idenburg SC, Aufdemkampe G, Wittink HM. The effect of physical exercise on cancer-related fatigue during cancer treatment: a meta-analysis of randomised controlled trials. Clin Oncol ( R Coll Radiol) 2010;22:208-221.

Zhu W, Timm G, Ainsworth B. Rasch calibration and optimal categorization of an instrument measuring women's exercise perseverance and barriers. Res Q Exerc Sport 2001;72:104-116. 